\title{
The challenge of restoring a fractured central incisor: a staged approach
}

\begin{abstract}
The restoration of a fractured central incisor is a major challenge to the clinician in his daily practice. An accurate anterior dental prosthetic must be suited for proper function, aesthetics, comfort and phonetics. It calls upon our knowledge of the smile design principles of proportion, symmetry, harmony and tooth morphology. Fortunately, nowadays, ceramic systems made it possible to restore effectively the color, shape, emergence profile, and incisal translucency. The purpose of this clinical report is to describe the treatment of fractured discoloured central incisors and to show the different guidelines to guarantee a natural mimicry using a zirconia based ceramic restoration.
\end{abstract}

Volume 9 Issue 4 - 2018

\author{
Hanen Boukhris \\ Department of Dental Medicine, University of Monastir, Tunisia
}

Correspondence: Hanen Boukhris, Department of Dental medicine, University of Monastir, Sousse, 4000, Tunisia, Tel +21654198974, Email boukhris I5@gmail.com

Received: May 08, 2018 | Published: July 25, 2018

Keywords: upper central incisor, dental trauma, esthetics, ceramics

\section{Introduction}

Dental traumas considered an increasing public health problem, unlike dental caries that have been declining over the last decades. The majority of dental injuries occur in the anterior region, affecting mainly the maxillary central incisors ${ }^{1}$ that can have significantly negative functional, esthetic and psychological impact. Prosthetic rehabilitation of traumatized anterior teeth would alter the entire appearance of the patient by providing them with a beautiful smile. The patient gains not only a positively improved appearance, but also a potential moral "boost" that acts positively on their mental health and self-esteem. ${ }^{2}$ In fact, thanks to the evolution of concepts, a new era of esthetic restorative dentistry, which is a combination of scientific principles and artistic abilities, has begun. There are many treatment options for fractured central incisor, and it is possible to restore function and esthetic using very conservative restorative techniques. ${ }^{2,3}$ However, esthetic dentistry can be a complex and demanding process. Multiple choices in dental materials, prosthetic designs and laboratory techniques, can be confusing, especially in the last 10 years, where the numerous choices available have been increasing the dentist's responsibilities, his role has been involving not only diagnosis and treatment planning but also communication and efficiency. Various predetermining factors play an important role in the evaluation and decision-making process of the treatment planning of each case. When a fractured central incisor must be restored, many restorative modalities are proposed from the less invasive to the most invasive, from direct composite restorations to full-coverage crowns. Clinical indication depends on the coronal dilapidation. In addition, many factors should be thoroughly determined before the actual treatment begins. It seems to be an easy task but many details must be carefully analyzed to achieve a natural mimicry of the maxillary central incisor restoration with the adjacent teeth. In the present paper, our case report describes a multi-disciplinary esthetic restoration of fractured central incisors.

\section{Case presentation}

\section{Diagnosis}

A 30-years-old male patient reported to the Department of Prosthodontics at CHU Farhat Hached of Sousse (Tunisia) in July 2016 with a chief complaint of restoring fractured maxillary central incisors (\#11; \#21) (Figure 1). He had suffered from a complicated crown fracture of the maxillary central incisors that occurred due to a domestic fall, leading to an esthetic liability. He had no medical history. He asked for an aesthetic, pleasing and natural appearing correction to improve his looks and smile.

Clinical and radiographic examination revealed that (Figure 1) (Figure 2):

1. The left central incisor was fractured in the middle-third of the crown, involving enamel and dentin fracture with pulp exposure. It was discoloured and the vitality test was negative.

2. The right central incisor was fractured at the mesial incisal angle.

Based on clinical and radiographic findings, various treatment options were discussed with the patient from direct composite restorations to full-coverage crowns. Patient was interested in aesthetical durable restoration.

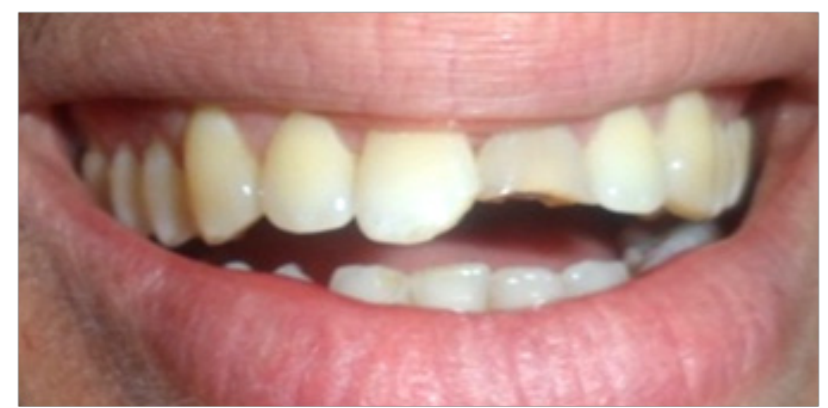

Figure I Facial view of initial situation.

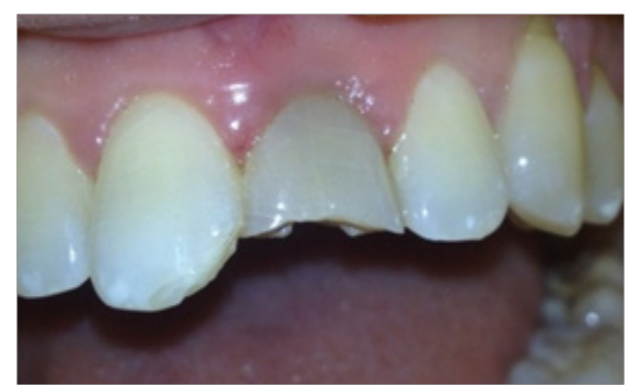

Figure 2 Intra oral views. 
We proposed to restore the fractured angle of the 11 with a direct composite resin restoration because of the satisfactory residual volume of coronal tooth structure. In the other hand, the treatment plan of the 21 included intentional root canal treatment followed by a definitive restoration made of metal-free crown. In such a clinical scenario, Zirconia is probably, the best option thanks to its semi-opaque aspect which mimics the adjacent opaque high value teeth. In addition, it helps us to mask the dyschromic aspect of the tooth.

\section{Clinical procedure}

First, the patient was recommended to take up initial periodontal preparation and to intensify oral hygiene before starting the rehabilitation process. Final dental restoration of the 11 using composite resin was performed in the first visit: After cleaning the tooth with pumice and water, hybridization of enamel and dentin was performed using a two-step total-etch system (Adper Single Bond 2; 3MESPE, Sumare, Brazil). A multilayer technique with freehand sculpting was used to build up the restoration (Anatomic stratification technique). An endodontic treatment was realized on the 21 under rubber dam isolation. An internal and an external tooth bleaching were performed in order to decrease the degree of dental discoloration (Figure 3) (Figure 4). There are certain recommendations, which had to be known to realize the preparation of a zirconia -based all ceramic restoration, we had to respect the guidelines of preparation design (no sharp angles, smooth margins ....). The preparation was achieved using flat and tapered diamond bur. Then, a complete arch impression was made with a silicone impression material (HydroC, Detax, Germany) (a combination of heavy -low and light silicon ). And, it was transferred to the laboratory to be casted (Figure 5). The shade was determined with a shade guide (Vitapan 3D Master-Vita). The provisional crown was elaborated by isomoulage technique using a silicon index and auto polymerized resin (Texton:SS White, Ce 0473, Prima Dental Group,England), it had to be precisely fitting and have highly polished margins. Finally it was cemented with temporary non eugenol cement (Temp Bond:Type I Class 1Ce 0086, Kerr, Italy). In laboratory, the working cast was scanned and framework was manufactured after step of computer-assisted design CAD/CAM indirect technique. Zirconia framework always requires a try-in step to confirm fit, insertion, retention, marginal integrity and mostly the space left for cosmetic ceramic (Fig. 6).Then, ceramic stratification was performed in the lab (Figure 7). A final try-in, of the zirconia ceramic crown was achieved again to verify esthetic, occlusion relationship and marginal integrity (Figure 8). Once validated, the zirconia crown was cemented using adhesive cement.
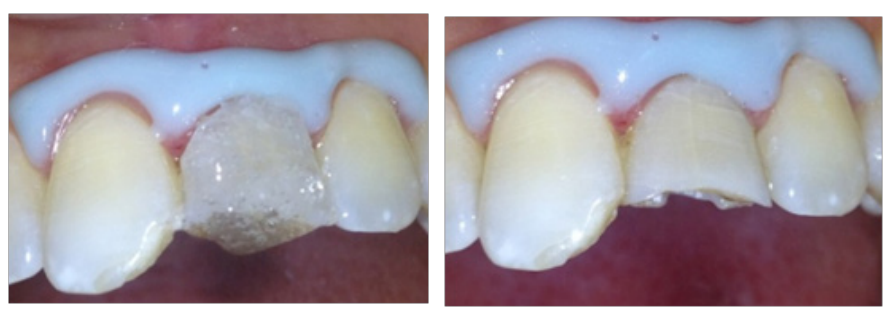

Figure 3 \& 4 During end after an internal and an external tooth.

\section{Discussion}

There are different restorative techniques used to treat central incisor fractures. The decision is based on the extent of tooth fracture. Currently, it is possible to restore function and esthetics using very conservative restorative techniques. ${ }^{2}$ For fractures located in dental enamel or in enamel and dentin without loss of much tooth structure, the use of composite resin is an excellent alternative. ${ }^{4,5}$ It is recommended to use a nanoparticles-based composite resin with the layering technique. ${ }^{5}$ Which allows restorations with nuances and shades of color similar to the adjacent teeth. However, good results are hard to achieve, this technique requires knowledge of the field of restorative material, knowledge of dental anatomy, and the manual skills to reproduce all the characteristics of the tooth. ${ }^{4}$ In clinical situations with great loss of coronal structure, the use of direct composite resins may not be the most appropriate therapeutic modality. ${ }^{6}$ Because the restoration probably will not support the masticatory efforts, mainly because of the insufficient area for bonding. ${ }^{6}$ In such cases, we prefer to use all-ceramic indirect restorations as they offer good aesthetic results that may be difficult to achieve with metal-ceramic systems. Nowadays, the improved mechanical properties of dental ceramics and the optical qualities of these materials have allowed the use of ceramics with esthetic predictability. ${ }^{7}$ Moreover, some types of ceramics, such as those based on Lithium Disilicate, have mechanical and micromechanical adhesion to the tooth structure through conditioning of the ceramics with hydrofluoric acid, a silane coupling agent, and subsequent bonding with resin cement. ${ }^{4}$ This makes retention of restorations possible, even when there is little coronal structure available. Other important features of the ceramics are surface smoothness and resistance to degradation. ${ }^{8}$ These characteristics enable color stability and integrity of the restorations over time. ${ }^{8}$ Moreover, these features contribute to the excellent response of gingival tissues, as it provides an environment similar to the enamel with reduced aggregation of bacterial biofilms. ${ }^{9}$ In complex cases of trauma with tooth fracture at or below the level of the crestal bone, we face a periodontal and restorative challenge. We should do crown lengthening in order to increase the clinical crown length to ensure an aesthetic restoration of the tooth without violating the biologic width. ${ }^{10,11}$ This procedure can be either surgical (by gingivectomy or apically positioned flap with or without bone recontouring.)or orthodontic. ${ }^{10-12}$ The main disadvantage of surgical crown lengthening is that supporting bone from adjacent teeth is removed to achieve an even contour, which can compromise their function. This procedure can also result in loss of the interdental papilla, opening of the embrasures, long clinical crowns and could compromise the aesthetics, which the clinician is striving to achieve. ${ }^{13,14}$ That is the reason why, the orthodontic forced eruption technique is best suited in cases where optimal aesthetics is required.

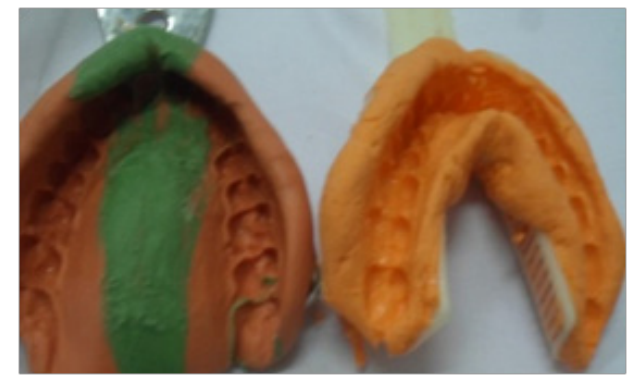

Figure 5 Arch impression.

Orthodontic tooth extrusion can be done as a slow or rapid extrusion (accompanied with a circumferential fiberotomy). ${ }^{10}$ Moreover, rapid extrusion is preferred to slow extrusion as it obviates the additional 
surgical procedure which is needed when a tooth is slowly extruded. ${ }^{10}$ The use of Zirconia based fixed prostheses can be well suited in such a clinical situation. It allows us to realize an intra-sulcular palatal margins which contraindicates the bonding procedure. ${ }^{15}$

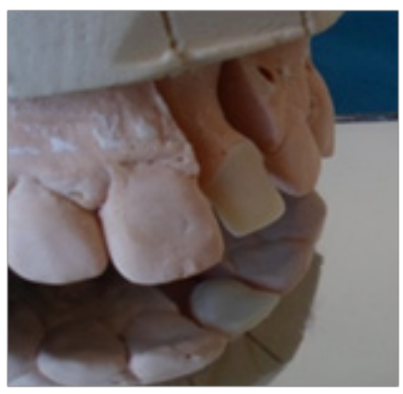

Figure 6 Zirconia frame.

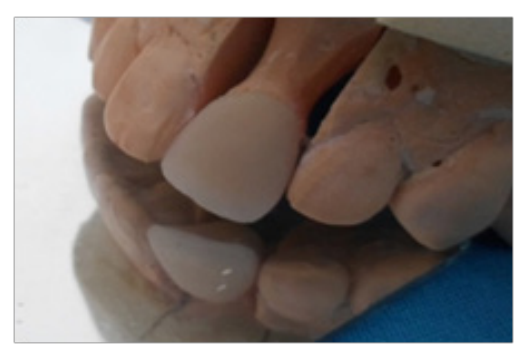

Figure 7 Final restoration.

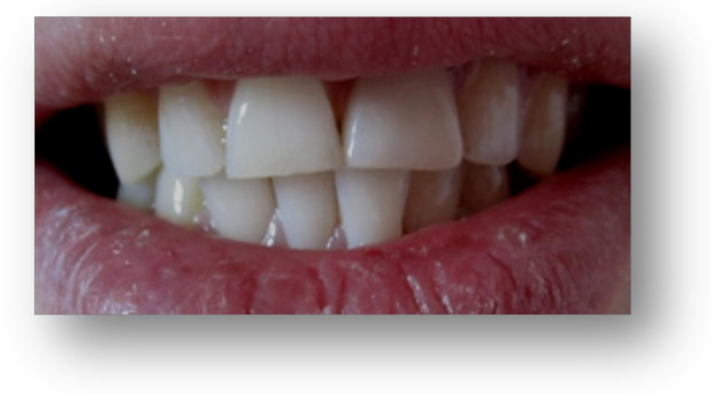

Figure 8 Final result.

\section{Conclusion}

Multiple treatment options are available for restoring a fractured central incisor. The clinician has to bear in mind the most important criteria which are the preservation of both aesthetic and function. The treatment depends also upon the extent of tooth fracture and also the clinician's preference. This multidisciplinary treatment requires the expertise of the endodontist, periodontist, orthodontist and prosthodontist. Then, the key to achieve success is based on Communication, patience, skills and artistic talent.

\section{Conflict of interest}

The author declares that there is no conflict of interest.

\section{References}

1. Marcenes W, Al Beiruti N, Tayfour D, et al. Epidemiology of traumatic injuries to the permanent incisors of 9-12-year-old schoolchildren in Damascus, Syria. Endod Dent Traumatol. 1999;15(3):117-123.

2. Gürel, Galip, Galip Gürel. The science and art of porcelain laminate veneers. London: Quintessence; 2003.

3. Castro MAM, Poi WR, Castro JCM, et al. Crown and crown-root fractures: an evaluation of the treatment plans for management proposed by 154 specialists in restorative dentistry. Dent Traumatol. 2010;26(3):236-242.

4. Anchieta, Rodolfo B, Rocha, et al. Recovering the function and esthetics of fractured teeth using several restorative cosmetic approaches. Three clinical cases. Dent Traumatol. 2012;28(2):166-172.

5. Sakai VT, Anzai A, Silva SM, et al. Predictable esthetic treatment of fractured anterior teeth: a clinical report. Dent Traumatol. 2007;23(6):371-375.

6. D'Arcangelo C, De Angelis F, Vadini M, et al. Fracture resistance and deflection of pulpless anterior teeth restored with composite or porcelain veneers. J Endod. 2010;36(1):153-156.

7. Chen YW, Raigrodski AJ. A conservative approach for treating young adult patients with porcelain laminate veneers. $J$ Esthet Restor Dent. 2008;20(4):223-238.

8. Pires-de-Souza Fde C, Casemiro LA, Garcia Lda F, et al. Color stability of dental ceramics submitted to artificial accelerated aging after repeated firings. J Prosthet Dent. 2009;101(1):13-18.

9. Aykent F, Yondem I, Ozyesil AG, et al. Effect of different finishing techniques for restorative materials on surface roughness and bacterial adhesion. J Prosthet Dent. 2010;103(4):221-227.

10. Rajendran, Maheaswari, Kshirsagar, et al. Crown lengthening by orthodontic forced eruption-a case report. International Journal of Current Research and Review. 2015;7(13):79.

11. Shyammohan A. Forced eruption: An adjunct to prosthodontic treatment planning. Indian J Stomatol. 2011;2(4):260-262.

12. Liudvikas Planciunas, Alina Puriene, Grazina Mackeviciene. Surgical lengthening of the clinical tooth crown. Stomatologija Baltic Dental and Maxiollofacial Journal. 2006;8:88-95.

13. Ayush Razdan Singh, Ruchita Verma. Crown lengthening versus forced eruption. Orthodontic Journal of Nepal. 2011;1(1):52-55.

14. Rob Veis. Forced eruption - Raising the root with gentle, conti-nous force in a coronal direction. The Practice Building Bulletin. 1993;1(9).

15. Raigrodski A. Managing the Challenge of Crowning the Single Central Maxillary Incisor. Journal of Esthetic and Restorative Dentistry. 2008;20(5):337-342.

\section{Acknowledgements}

None. 